\title{
Interactions between renal tubules and interstitium
}

\author{
A J Howie, C J Lote
}

\section{Introduction}

This review considers the physiological interactions between tubules and interstitial tissues in the kidney, and then their pathological interactions. Tubules and interstitium are so closely interrelated that they are often regarded as one, for instance in the term tubulointerstitial disease, although there is much more practical value in both physiology and pathology in consideration of each component separately. ${ }^{12}$

\section{Physiological interactions between renal tubules and interstitium}

Interstitial tissues and tubules have different functions in different parts of the kidney. ${ }^{1}$

\section{RENAL CORTEX}

In the cortex there is only a small amount of interstitium, and extracellular space and interstitial cells occupy only $8 \%$ of the cortical volume. In many parts of the cortex, tubules and capillaries are parallel to each other and their basement membranes are fused or in close proximity.

Renal interstitial tissues are the medium through which reabsorbate and secretions pass from the tubular lumen to the blood and vice versa. Fluid and solutes move across capillary walls by a combination of diffusion dependent on concentration gradients, and bulk flowthat is, filtration of water and those solutes able to cross capillary walls. Bulk flow is determined by the balance between capillary hydrostatic pressure that forces fluid out of the capillary and oncotic pressure due to plasma proteins that draws fluid into the capillary. Outside the kidney the capillary bed as a whole behaves so that there is an approximate balance between formation and reabsorption of tissue fluid.

By contrast, in the cortex the balance of forces is greatly in favour of capillary uptake. This is because the blood in peritubular capillaries has already passed through afferent arterioles, glomerular capillaries and efferent arterioles, and has a low hydrostatic pressure, but has a high oncotic pressure as a consequence of glomerular filtration of water and solutes but not albumin. There is efficient uptake of tubular absorbate from lateral intercellular spaces and across tubular cell membranes.
Leakage of albumin and other plasma proteins across glomerular basement membranes impairs the efficiency of this uptake by reducing peritubular capillary oncotic pressure and generating oncotic pressure in the tubular lumen. Expansion of cortical interstitium may be associated with disorders of tubular function, but there is a problem in deciding which process comes first. One effect of such expansion is likely to be reduced oxygenation of tubules. ${ }^{3}$

RENAL MEDULLA

The outer medulla has even less interstitium than the cortex, as its interstitium occupies only $5 \%$ of the volume. This minimises diffusional loss of solute from the deeper medullary interstitium, which is hypertonic, to the cortex, which is essentially isosmotic with plasma.

Deeper parts of the medulla have a large interstitial volume, at the papillary tip occupying $30 \%$ of the volume in the rat and $40 \%$ in the rabbit. ${ }^{4}$ The interstitium is gelatinous with large amounts of glycosaminoglycans. Its hypertonicity is responsible for water reabsorption from collecting tubules and is maintained by a dynamic equilibrium. Solute addition to the interstitium from the ascending limb of the loop of Henle is balanced by solute removal by longitudinal diffusion into the cortex and by the vasa recta, and by water entry from collecting tubules and vessels.

Exchanges occur between descending and ascending vasae rectae, and water leaves the descending limb and enters the ascending limb by countercurrent exchange. Oxygen and carbon dioxide also undergo countercurrent exchange in different directions, resulting in a low $\mathrm{PO}_{2}$ and a high $\mathrm{PCO}_{2}$ in the papillary interstitium. Cells in this region are able to use anaerobic metabolism but this can be impaired by a worsening of the hypoxia. ${ }^{35}$

\section{Pathological interactions between renal tubules and interstitium}

Structural abnormalities of tubules and interstitium are common in renal disease (figs 1 and 2) and are often associated with abnormalities of renal function such as reduced excretory function - that is, a fall in glomerular filtration 


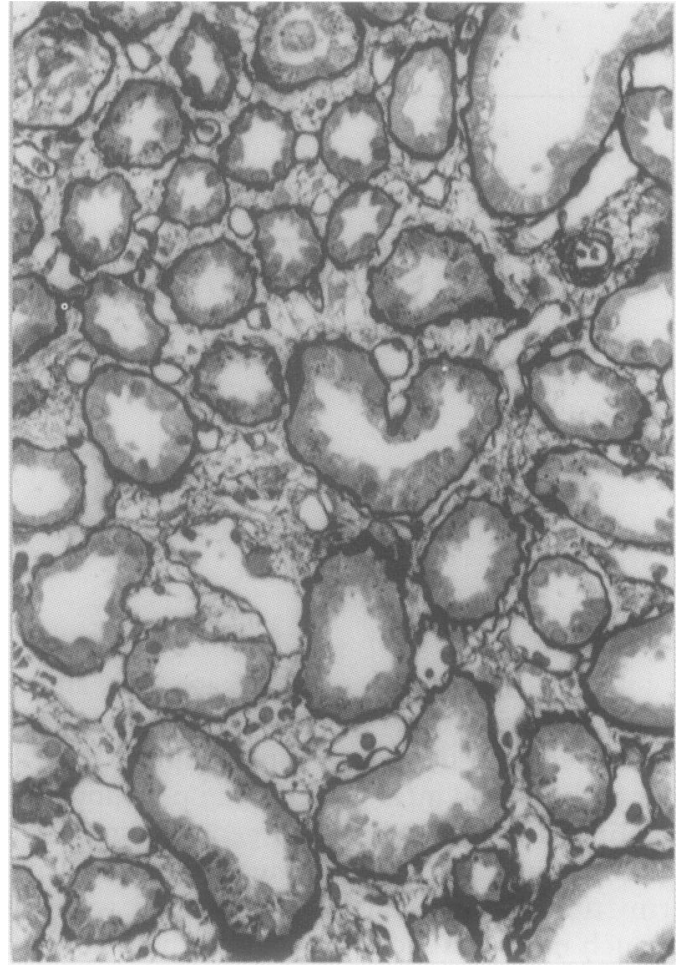

Figure 1 Interaction between cortical tubules and interstitium in acute renal failure due to a non-steroidal anti-inflammatory drug. Tubules are acutely damaged and there is interstitial oedema. Renal function recovered to normal. (Periodic acid-methenamine silver; original magnification $\times 200$.)

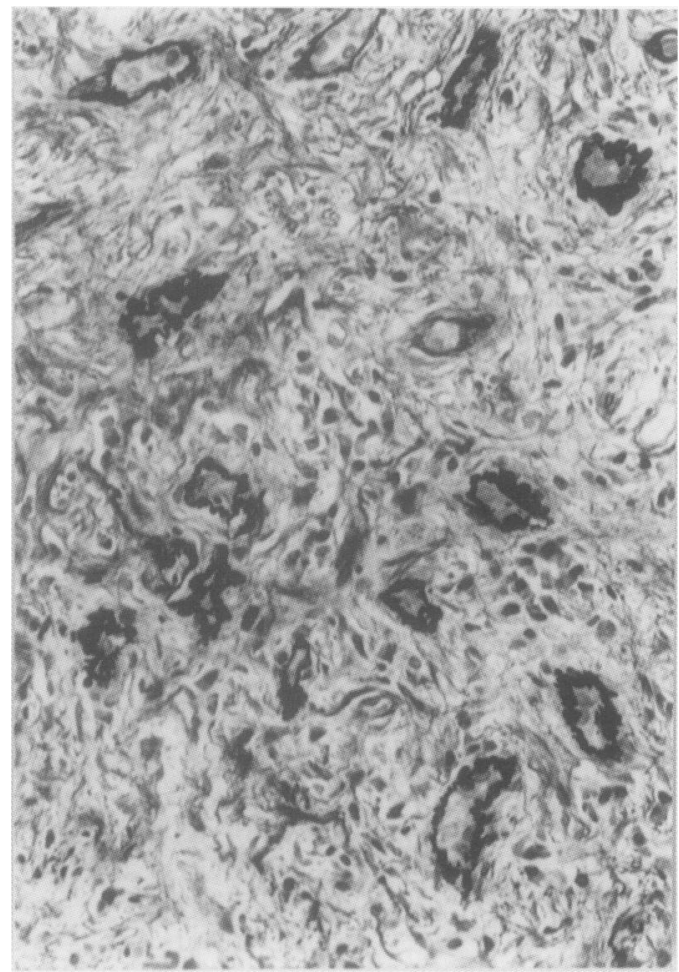

Figure 2 Interaction between cortical tubules and interstitium in chronic renal failure as a result of arteritis. Tubules are atrophic and there is interstitial fibrosis. Renal function did not recover. (Periodic acid-methenamine silver; original magnification $\times 200$.)

rate, shown clinically by a rise in plasma creatinine concentration. ${ }^{2}{ }^{6} \mathrm{~A}$ useful way to analyse pathological interactions is to consider tubular events affecting interstitium and vice versa.
FUNCTIONAL SIGNIFICANCE OF DISEASE IN TUBULES AND INTERSTITIUM

Correlations are observed in many renal disorders between impairment of renal excretory function and various measures of tubular and interstitial abnormalities. ${ }^{6-8}$ Problems that follow from these observations are whether tubular and interstitial changes always occur together, whether changes in one structure are more important in their effect on renal function or association with it, and whether tubular changes or interstitial changes are the primary event.

Although changes in tubules and interstitium usually occur together, there are a few exceptions, and these are important because they show that the state of tubules is more significant than the state of interstitial tissues in determining renal function or in association with it. ${ }^{2}$ For instance, there is no difference in renal interstitial inflammation and oedema between people with acute renal failure and those recently recovered from it, but there are differences in extent of loss of brush border in proximal tubules and in necrosis of tubular cells. $^{9}$

At first sight there is a paradox in that the glomerular filtration rate seems to be more closely related to structural changes in tubules than to changes in glomeruli. ${ }^{78}$ The explanation is complex and differs in different types of renal failure, but often the explanation is reduced renal blood flow, a feature of many types of renal failure. There can be diversion of blood from cortex to medulla and the reduced perfusion is sometimes severe enough to produce cortical necrosis. Tubules are sensitive to ischaemia and acute tubular damage is often a reflection of this renal hypoperfusion. ${ }^{310}$

TUBULAR EVENTS HAVING AN EFFECT ON INTERSTITIUM

Damaged tubules can release mediators that affect interstitial tissues and can also express antigens that have an immunological effect. ${ }^{11}{ }^{12}$ Not all tubular damage is necessarily followed by interstitial inflammation and fibrosis, and there are many complicated factors that determine the eventual outcome. ${ }^{13}$

\section{Causes of tubular damage}

Tubules can be damaged by ischaemia, toxins, infective agents, and immunological attack, and the cause of the damage is likely to have a major effect on the interactions between tubules and interstitium. One toxic factor that is now recognised is the presence of protein in the tubular lumen. ${ }^{14}$ Proteinuria is a feature of nearly every glomerular disorder and is also found late in the course of renal diseases that were not initially due to a glomerular disorder. This is because loss of glomeruli from any cause leads to hyperfiltration in surviving glomeruli, that eventually lose their ability to retain proteins in the circulation. ${ }^{15}$ The damaging effect on tubules of glomerular protein leakage is proportional to the severity and duration of proteinuria and can occur without obvious clinical abnormalities of excretory function. ${ }^{16}$ Protein is reabsorbed by proximal 
tubular cells and toxic effects of reabsorbed protein may be due to fatty acids bound to albumin rather than albumin itself, and to lipoproteins, complement components and metal containing proteins such as transferrin. ${ }^{12}$ Immunoglobulin light chains have a similar effect in myeloma, and so does haem in glomerular leakage of red blood cells or in intravascular haemolysis. ${ }^{12}$ Uptake of lipids by interstitial macrophages in prolonged proteinuria leads to the appearance of foamy interstitial cells. ${ }^{11}$

\section{Factors released by tubular cells that affect interstitium}

Tubular cells exposed to protein in the lumen or damaged in other ways can release endothelins. ${ }^{17}$ These have various effects on interstitial fibroblasts, inflammatory cells and blood vessels, in that fibroblasts are stimulated to proliferate and make collagen and other matrix proteins, macrophages are attracted and release cytokines, and blood vessels constrict. Endothelins may also stimulate tubular regeneration. ${ }^{17}$ The overall effect on tubules of release of endothelins depends upon the balance between damaging influences and the stimulus to recovery caused by influences to regenerate. This emphasises the complexity of the interaction between tubules and interstitium.

Other tubular effects on interstitium include interstitial fibrosis stimulated by factors from tubules such as cytokines, including platelet derived growth factor, and the influx of macrophages and $T$ lymphocytes, attracted by tubular release of such factors as interleukin- 6 and monocyte chemoattractant protein- $1 .{ }^{11-13}$ Loss of tubules leads to compensatory changes in surviving tubules. These have increased uptake of oxygen and increased production of reactive oxygen species, which in turn can cause further tubular damage and can induce interstitial fibrosis. ${ }^{18}$ Similar effects may follow generation of increased amounts of ammonia by surviving tubules. ${ }^{13}$

Tubular cells normally make collagens of basement membrane types, but when damaged may secrete collagens of other types, which contribute to interstitial fibrosis. ${ }^{13}$

\section{Antigens expressed by tubular cells that affect interstitium}

Tubules can have antigens not normally expressed if they contain drugs or infective agents, ${ }^{2}$ if they take up protein from the glomerular filtrate including products of glomerular damage such as basement membrane material, ${ }^{19}$ or if they are stimulated by cytokines. ${ }^{11}$ Tubules stimulated by interferon $\gamma$, from interstitial inflammatory cells, express class II major histocompatibility antigens, which allow them to act as antigen presenting cells to CD4+ $\mathrm{T}$ lymphocytes. Intercellular adhesion molecule-1 has expression enhanced by interferon $\gamma$ in tubular cells and this promotes adhesion of inflammatory cells. ${ }^{11}$ New antigens on tubular cells, antibodies directed against tubular basement membranes, and immune complexes deposited on tubular basement membranes attract immunocompetent interstitial cells and may lead to immune mediated tubular damage..$^{20}$ Release of normal tubular antigens such as Tamm-Horsfall protein can stimulate an antibody response, but there is doubt whether this contributes to further renal damage. ${ }^{21}$

\section{INTERSTITIAL EVENTS HAVING AN EFFECT ON} TUBULES

Interstitial inflammation can damage tubules, for instance by cytotoxic effects of CD8+ T lymphocytes, and can stimulate fibrosis, by release of factors from macrophages and $T$ lymphocytes, such as transforming growth factor $\beta$, interleukin- 4 and platelet derived growth factor. ${ }^{22}$ As well as these and factors from tubules, other chemicals can stimulate interstitial fibroblasts, including angiotensin 2, generated in the systemic circulation and in the kidney itself. ${ }^{23}$ Interstitial fibrosis is accompanied by a reduction in the peritubular capillary bed, and this and vasoconstrictive effects of agents such as endothelins may make tubules ischaemic and contribute to further tubular damage. ${ }^{1719}$

Although there are associations between interstitial changes and aspects of renal function, there is no immediately obvious mechanism by which the interstitium could control renal excretory function - that is, the glomerular filtration rate. ${ }^{8}$ Explanations have been suggested such as the obliteration of peritubular capillaries either reducing glomerular blood flow $^{19}$ or inducing glomerular enlargement and subsequent segmental and global sclerosis. ${ }^{13}$ The more likely explanation is that interstitial changes are associated with tubular changes, and that these have a more intimate but complex relation with renal excretory function. $^{3610}$

Tubular atrophy is accompanied by interstitial fibrosis and a chronic inflammatory infiltrate. $^{2}$ This seems the mechanism, almost physiological, by which there is loss of a tubule that is no longer functional, for instance if its glomerulus is lost by ischaemia or destroyed by glomerulonephritis. The interstitial events in these and other disorders, especially acute interstitial nephritis, seem to be initiated by tubular events although they can perpetuate the tubular damage and lead to tubular atrophy.

There is interest in possible ways of preventing interstitial fibrosis, in the hope that this will preserve or improve renal excretory function. ${ }^{24}$ More important for the preservation or recovery of function are possible ways of helping tubules to return to normal, as acutely damaged tubules have the potential to recover but atrophic ones are beyond recovery. ${ }^{25}$ This is a similar idea to the value of the activity and chronicity scores in assessment of the prognosis of lupus nephritis. ${ }^{26}$

\section{Summary}

Renal tubules and interstitium have close physiological associations. Changes in both are often seen in renal disease. Damaged tubules can attract inflammatory cells and stimulate interstitial fibrosis, but do not always do so. Interstitial inflammation can damage tubules 
and can also stimulate fibrosis, and is probably always initiated by tubular events. Interstitial and tubular abnormalities are closely associated with changes in renal excretory function, but tubular events are more important. A main determinant of the outcome of renal disease is whether tubules can recover, not the extent of interstitial changes. If tubules are atrophic, they will not recover and renal function will be permanently impaired.

1 Lote CJ. Principles of renal physiology. 3rd edn. London Chapman and Hall, 1994.

2 Howie AJ. Interstitial nephritis. In: Anthony PP, MacSween RNM, Lowe D, eds. Recent advances in histopathology. Vol 17. Edinburgh: Churchill Livingstone. In press.

3 Lote CJ. Renal physiology and acute renal failure. Curren Anaesthesia and Critical Care 1992;3:124-32.

4 Knepper MA, Danielson RA, Saidel GM, Pust RS. Quantitative analysis of renal medullary anatomy in rats and rabbits. Kidney Int 1977;12:313-23.

5 Neely JR, Grotyohann LW. Role of glycolytic products in damage to ischemic myocardium. Circ Res 1984;55:816-24.

6 Howie AJ, Gunson BK, Sparke J. Morphometric correlates of renal excretory function. $\mathcal{f}$ Pathol 1990;160:245-53.

7 Risdon RA, Sloper JC, de Wardener HE. Relationship between renal function and histological changes found in renal biopsy specimens from patients with persistent glomerular nephritis. Lancet 1968;ii:363-6.

8 Bohle A, Mackensen-Haen S, Gise H. Significance of tubulointerstitial changes in the renal cortex for the excretory function and concentration ability of the kidney: morphometric contribution. Am $₹$ Nephrol 1987;7:421-33.

9 Solez K, Morel-Maroger L, Sraer J. The morphology of "acute tubular necrosis" in man: analysis of 57 renal biopsies and a comparison with the glycerol model. Medicine 1979;58:362-76.

10 Trueta J, Barclay AE, Daniel PM, Franklin KJ, Prichard MML. Studies of the renal circulation. Oxford: Blackwell Scientific Publications, 1947.
11 Cameron JS. Tubular and interstitial factors in the progression of glomerulonephritis. Pediatr Nephrol 1992;6:292 303.

12 Fine LG, Ong ACM, Norman JT. Mechanisms of tubulo-interstitial injury in progressive renal diseases. Eur $\mathcal{f}$ Clin Invest 1993;23:259-65.

13 Dodd S. The pathogenesis of tubulointerstitial disease and mechanisms of fibrosis. Curr Top Pathol 1995;88:51-67.

14 Benigni A, Zoja C, Remuzzi G. The renal toxicity of sustained glomerular protein traffic. Lab Invest 1995;73. $461-8$.

15 Howie AJ. Segmental sclerosing glomerular lesions. Pediatr Nephrol 1993;7:370-4.

16 Howie AJ, Rowlands DC, Reynolds GM, Barnes AD Measurement of proliferation in renal biopsy specimens: evidence of subclinical tubular damage in the nephrotic syndrome. Nephrol Dial Transplant 1995;10:2212-18.

17 Ong ACM. Tubulointerstitial actions of endothelins in the kidney: roles in health and disease. Nephrol Dial Transplant 1996;11:251-7.

18 Nath KA, Fischereder M, Hostetter TH. The role of oxidants in progressive renal injury. Kidney Int 1994; 45(Suppl 45):S111-15

19 Bohle A, Wehrmann M, Mackensen-Haen S, Gise H, Mickeler E, Xiao TC, et al. Pathogenesis of chronic renal failure in primary glomerulopathies. Nephrol Dial Transplant 1994; 9(Suppl 3):4-12.

20 Neilson EG. Pathogenesis and therapy of interstitial nephritis. Kidney Int 1989;35:1257-70.

21 Papanikolaou G, Arnold AJ, Howie AJ. Tamm-Horsfall protein in reflux nephropathy. Scand $\mathcal{F}$ Urol Nephrol 1995;29: $141-6$

22 Strutz F, Neilson EG. The role of lymphocytes in the progression of interstitial disease. Kidney Int 1994; 45(Suppl 45): S1 106-10.

23 Egido J. Vasoactive hormones and renal sclerosis. Kidney Int 1996;49:578-97.

24 Strutz F, Muller GA. On the progression of chronic renal disease. Nephron 1995;69:371-9.

25 Pirani CL. Evaluation of kidney biopsy specimens. In Tisher CC, Brenner BM, eds. Renal pathology with clinical and functional correlations. Philadelphia: Lippincott, 1989:24-8.

26 Kashgarian M. Lupus nephritis: lessons from the path lab. Kidney Int 1994;45:928-38. 